\section{BARN OWLS \\ Catching Mice}

from our Animal Behaviour Correspondent

BARN owls (Tyto alba) can successfully catch mice in total darkness; they rely on the noise the mouse makes as it rustles through leaves to guide them to their prey. R. S. Payne (J. Exp. Biol., $54,535 ; 1971)$ has demonstrated that the owls can do this even in the absence of other clues as to mouse's whereabouts, such as its smell or the heat given off by its body, and his experiments have shown that the owls are remarkably accurate in both the horizontal and vertical localization of a sound source.

He induced owls to fly towards a loudspeaker that was emitting mouselike rustles and gauged their accuracy by measuring the distance between the centre of the loudspeaker and the centre of the imprint which the owls' talons made on impact with plasticine surrounding the loudspeaker. This method revealed that the owls seemed to need frequencies above $8.5 \mathrm{kHz}$, because when these frequencies were removed from the sound source, the birds were much less accurate.

Regardless of its position when it first hears a mouse rustle, an owl always turns to face the mouse and then must have one further sound before it can strike successfully. Payne suggests that this is because the owl localizes a complex sound source by trying to make all frequencies as loud as possible in both ears and would then automatically come to face the source of the sound. To investigate this, Payne placed a microphone in the head of a dead owl at the site of the (now removed) eardrum and played sounds of constant intensity and frequency through a loudspeaker, recording the response of the microphone as the position of the loudspeaker was varied. In this way, he was able to construct a map of "isointensity" lines for each frequency, showing the position of "highs" (places

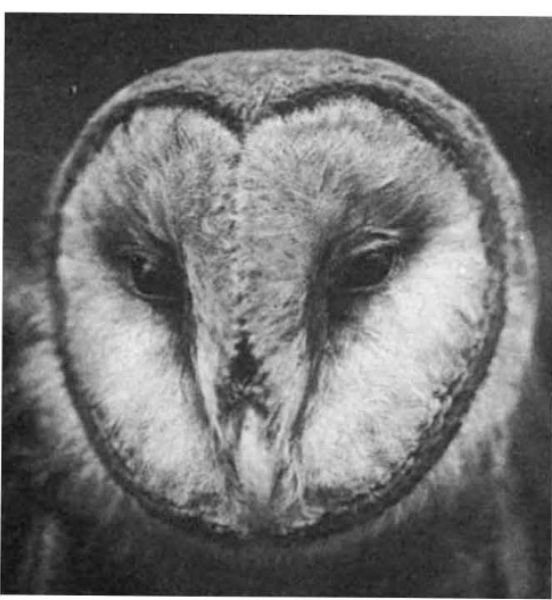

Barn owl (photo: Zoological Society of London). from where the sound was.loud) and "lows" in the space surrounding the ear. The owl's sensitivity was found to rise sharply to a maximum along the line of sight. A low in one ear always corresponded to a direction of good hearing for the other ear, and this was interpreted as a special adaptation to sound localization, because it would have the effect of sharpening the frontally directed regions of maximum sensitivity.

Comparisons of positions at which regions of maximum and minimum sensitivity for a given frequency occurred in the vertical plane revealed that in the right ear such regions are roughly
10-15 degrees higher than in the left ear, presumably as a result of the marked asymmetry of the external ears. A sound which was loud to the right ear could therefore be soft to the left. If the owl then tilted its head in the vertical plane, the relative loudness of the sound in the two ears would change, and this too seems to be an adaptation to reduce ambiguity about the position of a sound.

One of the most characteristic features of an owl, the heart-shaped face, may also be connected with its hearing ability; it may act as a parabolic reflector to catch sound.

\title{
Repairing Mispairing of DNA
}

IN next Wednesday's Nature New Biology a simple observation is described which may have profound genetical implications. Using the multicellular alga, Ulva mutabilis, Fjeld has produced evidence which lends support to the master-slave hypothesis of undirectional correction of mispairing of DNA and also gives an indication of the reasons for only complete mutations being found after ultraviolet irradiation.

It seems surprising that Ulva mutabilis has not been used more frequently for genetic studies because individual cells of this haplo-diplontic alga can be induced to form gametes which will germinate parthenogenetically giving haploid plants. Development starts as a single filament of cells which later becomes tubular, and the characteristic plant form is assumed by elongation of the basal cells to form the stem and the continued division of cells at the apex to form the blade. Chimaeras can be induced by irradiation of young algal germlings and Fjeld has found that there are two kinds which occur in equal numbers; one is genetic so that the gametophytic progeny also show the chimaeric characteristic and the other is merely phenotypic and the gametophytes appear wild type. The chimaeras examined were those with a basal part very like wild type but with a narrow protuberance at the apex which developed into a long ribbon, and those with a bulge somewhere on the blade.

In twenty-six plants with this first type of alteration the apical protuberance did not develop very far, and when the cells of these plants were induced to form gametes the change proved to be merely phenotypic. The apical protuberance on a further twenty-three plants, however, continued development into a long ribbon and analysis of these plants showed that the change in this instance was genetic. Those plants which responded to the ultraviolet light by producing a bulge on the blade also showed the genetic and phenotypic chimaeras to be produced in equal numbers.

It is easy to understand how the genetic alterations can be induced by ultraviolet light, but the nature of the temporary phenotypic alteration is more puzzling, and Fjeld's suggestion that this lies at the level of the gene is interesting. His explanation centres around the fact that only one strand of the DNA is involved in transcription and a mutational event occurring in this strand would be transcribed into an altered mRNA. This cell and any immediate descendants would thus be channelled into an abnormal developmental path giving rise to the chimaeras. Fjeld then goes on to suggest that if the strand which is being transcribed is corrected, using the other strand as template, a phenocopy appears, for all the gametophytic progeny from these cells, in which the abnormal development has been corrected, will be wild type. Up to this point his explanation means that the ratio of genetic to phenotypic chimaeras would be $2: 1$, for the probability of mutation and correction would be the same in both strands. Half of the mutations in the transcribed strand will thus revert to wild type and will register as phenotypic chimaeras, although if the same type of event occurs in that DNA strand which is not transcribed the mutational event will be unnoticed. Only mutations in this strand which do not revert and which register as genetic chimaeras will be recorded so that there would be an imbalance in favour of genetic changes.

There are many implausible ways of explaining away the discrepancy, but Fjeld's ideas are quite attractive for he chooses to believe that the transcribed strand is always corrected against the other strand but not vice versa. Mutations in the transcribing strand will thus always give rise to the phenocopies, whereas mutations in the non-transcribing strand will produce the true genetic alterations. The expected ratio will then be $1: 1$. 